\title{
Five Open Problems in Quantum Information Theory
}

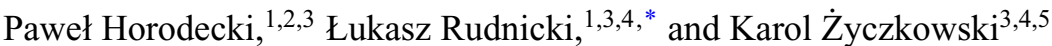 \\ ${ }^{1}$ International Centre for Theory of Quantum Technologies (ICTQT), University of Gdańsk, Gdańsk 80-308, \\ Poland \\ ${ }^{2}$ Faculty of Applied Physics and Mathematics, Technical University of Gdańsk, Gdańsk 80-952, Poland \\ ${ }^{3}$ National Quantum Information Centre (KCIK), University of Gdańsk, Poland \\ ${ }^{4}$ Center for Theoretical Physics, Polish Academy of Sciences, Aleja Lotników 32/46, Warsaw 02-668, Poland \\ ${ }^{5}$ Institute of Theoretical Physics, Jagiellonian University, Kraków 30-348, Poland
}

(Received 21 December 2020; revised 1 December 2021; published 3 March 2022)

\begin{abstract}
We identify five selected open problems in the theory of quantum information, which are rather simple to formulate, are well studied in the literature, but are technically not easy. As these problems enjoy diverse mathematical connections, they offer a huge breakthrough potential. The first four concern existence of certain objects relevant for quantum information, namely a family of symmetric informationally complete generalized measurements in an infinite sequence of dimensions, mutually unbiased bases in dimension six, measurements saturating multiparameter Cramér-Rao bound and bound entangled states with negative partial transpose. The fifth problem requires checking whether a certain state of a two-ququart system is two-copy distillable.
\end{abstract}

DOI: 10.1103/PRXQuantum.3.010101

\section{INTRODUCTION}

Roman Stanisław Ingarden, one of the founding fathers of the field, wrote in 1975: "The aim of the present paper was only to give a general formulation of the quantum-information theory of the Shannon type. The theory requires further investigations and mathematical development." At the time this paper Quantum information theory [1] was published, exactly 45 years ago, it was difficult to predict that such a piece of research in mathematical physics could inspire a vast new field of science and trigger a remarkable progress in experimental physics and yield numerous applications.

Indeed, the field of quantum information (see Refs. $[2,3])$ with its cornerstones of pioneering discoveries of quantum money [4], quantum cryptography [5,6], quantum dense coding [7], quantum teleportation [8], quantuminformation compression [9], and quantum computing [10-12] has visibly matured recently, therefore, more and more often we hear and read about quantum technologies.

*lukasz.rudnicki@ug.edu.pl

Published by the American Physical Society under the terms of the Creative Commons Attribution 4.0 International license. Further distribution of this work must maintain attribution to the author(s) and the published article's title, journal citation, and DOI.
The latter aim at turning famous theoretical concepts such as quantum cryptography into fully operational devices. These applications, based on "standard" technologies developed so far, possess essential functionalities solely operating on quantum principles.

Along the course of the, so-called, second quantum revolution, experimental efforts are mostly directed towards solutions to practical problems, such as mitigation of the noise and decoherence effects, or scalability. Therefore, a discussion of perspectives within experimental quantum information could certainly focus on new techniques allowing for a better protection and control of quantum systems.

On the theory side we observe a similar tendency. Current research focus is on optimization of theoretical protocols and experimental schemes, as well as discussion of practical limitations of the techniques developed. An example of a very recent, beautiful result [13] from the field of quantum metrology can serve us the purpose of illustrating the above trend. While it is known that, so-called, superresolution techniques [14] allow one to increase the precision beyond that of typical diffractionlimited direct imaging, robustness of this method is not fully understood. In Ref. [13], a scheme based on intensity measurements involving spatial mode decomposition has been scrutinized against experimental noise stemming from the crosstalk between the modes used. Deterioration of the quantum superresolution benefits has been 
found. Based on the above example it is easy to imagine a perspective article devoted to theoretical quantum information, pointing areas and problems, within all the pillars of quantum technologies [15], which require further attention.

In our work, however, we take a perspective visibly different from that described above. Being aware of the currently relevant, particular challenges of theoretical quantum information, we ask ourselves whether there is still room for ground-breaking, though not completely unexpected, developments. To let this question have an affirmative answer, we identify open problems with such a breakthrough potential [16]. We require the problems to be as follows:

(a) well studied and extensively covered by the topical literature, so that a convincing evidence of their importance exists;

(b) technically hard, so that they require methodology beyond the toolbox available at the moment;

(c) universal and with a rich mathematical underpinning, so that they are not associated with narrowly defined platforms or protocols.

The first criterion assures the recognition, the proposed problems have gained. Being well studied implies that the problems have a long history deeply immersed in the field of (theoretical) quantum information, therefore, a future solution shall expand the base of the field, rather than one of its distant branches. The second criterion, beyond offering an explanation why the problems still remain open, pertains to the future impact of the solutions. Presumably advanced techniques necessary to tackle the problems, perhaps not yet recognized or even established, will likely make an impact beyond their initial niche. Finally, since we observe the tendency towards specialization and narrowing of the research conducted, trends that are essential at a stage where initially broad concepts are being turned into concrete devices, we look for breakthrough theoretical discoveries beyond this modus operandi. Likely, only unexpected solutions to problems, which are not associated with a particular setup, can influence the whole field of quantum information.

In our tight selection of the open questions to be offered as a future inspiration and guideline for theoretical research, we restrict our attention to five concrete problems. Why five? As it turns out, the number six, if used to set the dimension of the Hilbert space, is still insufficiently well understood in the context of quantum information. While the above justification gives as good a reason as any other reason, the first two problems described below are in fact associated with symmetric configurations in discrete Hilbert spaces, and the second one is to some extent concerned with this special dimension.

\section{DISCRETE STRUCTURES IN THE HILBERT SPACE}

The space of pure quantum states of a fixed dimension [17] $N$ is isotropic - no quantum state is "more equal than others." However, this property does not exclude existence of complex and at the same time well-organized structures inside the Hilbert space, e.g., particular constellations of quantum states with prescribed properties. It is easy to imagine, that each structure of such kind nurtures a potential for quantum-information protocols such as those used in error correction, or particular experimental tasks, such as quantum tomography. Readers familiar with the background of theoretical quantum information will likely recognize that mutually unbiased bases (MUBs) and symmetric informationally complete positive operator-valued measures (SIC POVMs) provide natural examples of such structures. Intriguingly, in both cases there is an important missing piece of the puzzle, which we now turn into an open problem.

The Fire Chief appearing in the middle of The Bald Soprano, a first play written by Eugéne Ionesco, says "I should like to remove my helmet, but I haven't time to sit down" [18]. After saying this he immediately "sits down without removing the helmet." In one aspect of this perspective we are going to follow the same strategy. While we have just declared that the number of proposed problems shall be lower than six, in the Appendix we describe the sixth problem, extending the discussion of the current section to cover the third constellation, perhaps better known in classical considerations (therefore, we introduce it in more detail), namely the Latin squares (LSs). We have believed this problem would remain unsolved for a longer time, however, this turns out not to be the case anymore [19].

Following the common word of wisdom saying that the proof of the pudding is in the eating we cut here the general discussion and immediately pose the problems associated with the two constellations and structures mentioned above.

\section{A. Existence of SIC POVMs}

Problem 1. Construct SIC POVMs in an infinite sequence of dimensions, $N_{1}, N_{2}, N_{3}, \ldots$

Setup. A symmetric informationally complete positive operator-valued measure [20,21] associated with an $N$ dimensional complex Hilbert space $\mathcal{H}_{N}$ is given by a set of $N^{2}$ vectors $\left|\psi_{j}\right\rangle \in \mathcal{H}_{N}$ satisfying the following overlap relations:

$$
\left|\left\langle\psi_{j} \mid \psi_{k}\right\rangle\right|^{2}=\frac{N \delta_{j k}+1}{N+1}, \quad j, k=1, \ldots, N^{2} .
$$

This set defines a generalized quantum measurement capable to extract complete information concerning any density 


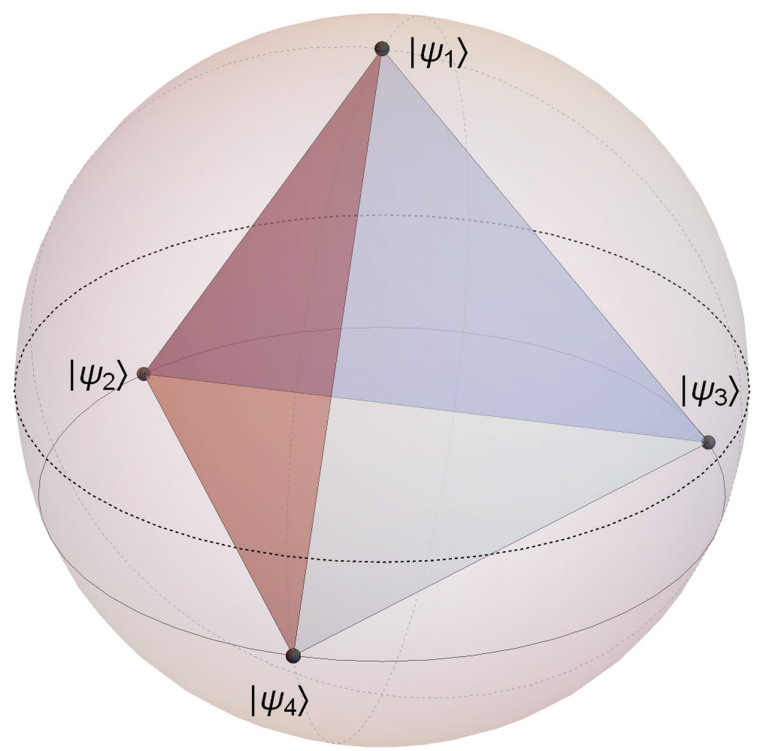

FIG. 1. Four pure states $\left|\psi_{i}\right\rangle$ span a regular tetrahedron inscribed in the Bloch sphere and lead to a single-qubit symmetric informationally complete measurement (SIC POVM) for $N=2$. Can you find $N^{2}$ pure states of size $N$ such that the corresponding projectors form a simplex inscribed into the set of quantum states of a given order $N$ ?

matrix of order $N$ described by $N^{2}-1$ real parameters. Moreover, such a constellation of $N^{2}$ projectors onto pure states forms a simplex inscribed in the entire $N^{2}-1$ dimensional set of density matrices of order $N$-see Fig. 1 for an $N=2$ example. For an accessible guide to the SIC problem in low dimensions consult Ref. [22].

Motivation. From a mathematical point of view, we ask about the maximal set of complex equiangular lines [23] in a given dimension $N$. From a physical perspective one looks for a scheme of an optimal quantum measurement of an arbitrary size $N$, distinguished by the fact that the number of projector operators is the minimal possible required to gather complete information concerning the analyzed state. Solving the SIC existence problem for any dimension will significantly contribute to our understanding of the geometry of the set of quantum states [24].

According to the 1999 dated conjecture by Zauner [20], for any dimension $N$ there exists a fiducial vector, such that all remaining $N^{2}-1$ elements of the desired SIC can be obtained by acting on it with unitary matrices representing elements of the Weyl-Heisenberg group.

Numerical solutions obtained in 2004 for all dimensions up to $N=45$ (Renes et al. [21]) were extended in 2010 by Scott and Grassl [25] to $N \leq 67$. Further results from 2017 included dimensions $N \leq 121$, Scott [26] and $N \leq 151$, Fuchs et al. [27]. In 2020 numerical solutions were known for $N \leq 193$ and also for $N=204,224,255,288,528,725,1155,2208$ (Grassl [28]). Analytical solutions are known for $N \leq 53$
$[21,25,26,29]$, and several other dimensions, including $N=57,61-63,65,67,73,74,76,78-80,84,86,91,93,95$, 97-99, 103, 109, 111, 120, 122, 124, 127, 129, 133, 134, 139, $143,146,147,151,155,157,163,168,169,172,181-183$, 193, 195, 199, 201, 228, 259, 292, 323, 327, 364, 399, 403, 489, 844, 1299 — see Refs. [28,30,31]. Recently, new solutions have been found in dimension $N=487,628,787$, 964, 1027, 1228, 1447, 1684, 1852, 2404, 2707, 4099, 5779,19603 , and 39604 [32]

However, in spite of a considerable research effort [3337], the general conjecture of Zauner remains unproven. Finding a family of SICs in any infinite sequence of dimensions could become a decisive step in this direction. Furthermore, let us emphasize inspiring connections to some major open questions in algebraic number theory, including a key part of the twelve problem of Hilbert [38-41].

\section{B. MUBs in dimension six}

Problem 2. Construct a set of at least four mutually unbiased bases in dimension six or prove that there are no seven MUBs in $\mathcal{H}_{6}$.

Setup. Consider a set of $K$ bases $\left\{\left|\psi_{i}^{m}\right\rangle\right\}(1 \leq m \leq K$, $1 \leq i \leq N)$ in $N$-dimensional complex Hilbert space $\mathcal{H}_{N}$, so that all vectors in each basis are orthogonal, $\left\langle\psi_{i}^{m} \mid \psi_{j}^{m}\right\rangle=$ $\delta_{i j}$. These bases are called mutually unbiased if any two bases are unbiased, which means

$$
\forall_{i, j} \quad\left|\left\langle\psi_{i}^{m} \mid \psi_{j}^{n}\right\rangle\right|^{2}=\frac{1}{N}, \quad m \neq n .
$$

It is relatively easy to show that there exist no more than $N+1$ MUBs in $\mathcal{H}_{N}$. Moreover, for any $N \geq 2$, there exist at least three MUBs (see Fig. 2 for an $N=2$ example). If the dimension $N$ is a prime number or a power of a prime, $N=p^{k}$, there exists a complete set of $N+1$ MUBs $[42,43]$. This implies that for a composite dimension represented by a product of powers of primes, $N=p_{1}^{k_{1}} \ldots p_{m}^{k_{m}}$, with $p_{1}^{k_{1}} \leq p_{2}^{k_{2}} \leq \cdots \leq p_{m}^{k_{m}}$, there exist (at least) $p_{1}^{k_{1}}+1$ MUBs [20,44]. It is also known that if one finds $N$ MUBs in dimension $N$ the last $(N+1)$ th unbiased basis also exist [45], so the maximal number of existing MUBs is either equal to $N+1$, or it is less or equal to $N-1$.

Several methods to construct MUBs are known $[44,46$, 47] and all solutions for dimensions 2-5 are classified [48]. If $N$ is a power of a prime, various properties of a complete set of $N+1$ MUBs are already understood [49-53], but otherwise the number of existing MUBs remains unknown [54-56]. In particular, for $N=6$ a complete set would consist of seven MUBs, but to date only solutions containing three bases were found [57-67]. It is however known that if a complete set of seven MUB exists, it cannot contain a triple of product bases $[68,69]$. 


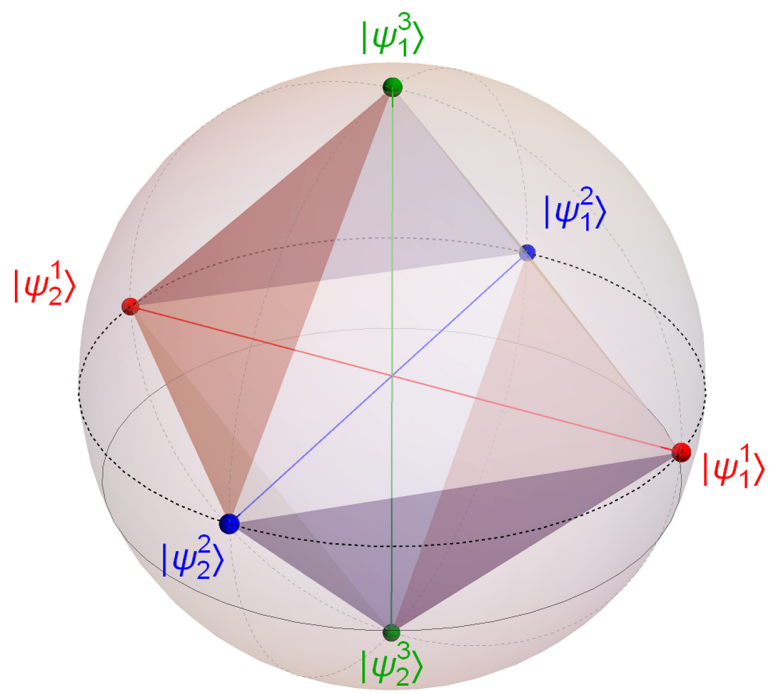

FIG. 2. Three eigenbases of Pauli matrices $\sigma_{x}, \sigma_{y}, \sigma_{z}$ span an octahedron inscribed in the Bloch sphere and form a set of three mutually unbiased bases for $N=2$. There exist four, five, and six MUBs in dimensions $N=3,4$ and 5, respectively. How many MUBs exist for $N=6$ ?

Any unitary matrix that relates two unbiased bases, $U_{i j}=\left\langle\psi_{i}^{m} \mid \psi_{j}^{n}\right\rangle$, belongs to the set of complex Hadamard matrices. This set consists of unitary matrices of order $N$, such that all its entries have the same squared modulus, $\left|U_{i j}\right|^{2}=1 / N$. Interestingly, the set of complex Hadamard matrices is again fully characterized [70-72] up to $N=5$. As several new complex Hadamard matrices of order $N=$ 6 were discovered a decade ago $[73,74]$, it was tempting to expect that they could lead to certain sets of four MUBs in this dimension [64]. However, to date the maximal number of MUBs for $N=6$ remains three, even though for larger dimensions further connections between Hadamard matrices and MUBs were found [75].

Motivation. On the one hand, finding a complete set of MUBs in dimension 6 would yield an optimal scheme of orthogonal quantum measurement in this dimension. More importantly, deciding whether such a configuration exists has significant implications for foundations of quantum theory, as to date our understanding of basic properties of finite-dimensional Hilbert spaces is not complete. On the other hand, a possible nonexistence result is of a considerable mathematical interest, as it would provide further arguments that the number 6 - the smallest product of two different primes - is indeed very special and "less equal than others." Let us emphasize here that there is no affine plane of order six and there are no orthogonal Latin squares of order six, which inspired the problem discussed in Appendix A. Research on the MUBs reveals further intricate links between foundations of quantum theory and several fields of mathematics, including Galois rings, group theory, combinatorics, finite fields, and projective geometry [76-84].

\section{Further perspectives I}

The two problems discussed above concern finite-dimensional Hilbert spaces. However, the notion of MUBs (or mutually unbiased measurements-MUMs) is also present in experimentally relevant setups involving continuous [85] or coarse-grained [86] systems. While in the continuous case we maximally have three MUBs [85], in the coarse-grained scenario [87] the situation is much more elaborate. Interestingly, the special dimension $N=6$ is not at all distinguished in the coarse-grained setting, since the systems of even dimension behave like the continuous ones (no more than three MUMs) - only odd dimensions nurture potential for more [87]. Whether this fact is connected to the conundrum of $N=6$ for discrete systems remains and open question at the moment.

Real Hadamard gates play a key role in numerous schemes of quantum-information processing. More general, complex Hadamard matrices are instrumental in Problem 2 concerning MUBs, but they also become linked [88] to Problem 1 on SICs. These matrices do exist in any dimension - as for any dimension $N$ we can write down the Fourier matrix $F_{N}$-in contrast to real Hadamard matrices [55]. They were constructed by Sylvester more than 150 years ago [89], but it was Hadamard who first showed [90] that such matrices do not exist unless $N=2$ or $N=4 k$. The celebrated conjecture due to Paley [91] that they do exist for all dimensions not excluded by Hadamard is now confirmed [92] up to $N=664$. The point is that finding a solution for $N=4 k$ gives us no clues, whether a real Hadamard matrix exists for $N^{\prime}=4 k+4$. We encounter a similar situation in Problem 1, as finding SIC for a given $N$ sheds no light into the existence problem in dimension $N+1$. However, it is straightforward to construct an infinite family of Hadamard matrices in dimension $N=2^{m}$ by tensor product, while we are still in search for a family of SICs in an infinite sequence of dimensions. Knowing a SIC configuration for a certain dimension $N$ it might be easier to look for another one in dimension $N(N-2)$, but such a SIC dimension tower $[35,36]$ remains, to date, of a finite size only.

The first two problems deal with pure quantum states from a single space $\mathcal{H}_{N}$, while the last one requires consideration of at least its two copies, $\mathcal{H}_{N} \otimes \mathcal{H}_{N}$. Such a space with a tensor-product structure corresponds to a physical system in which two subsystems can be distinguished. This construction allows one to introduce product states and entangled states, including the generalized Bell state (A1).

\section{QUANTUM METROLOGY}

In the Introduction we pointed towards current trends and practical problems considered in quantum metrology, such as those relevant for superresolution $[13,14,93,94]$. Now, in accordance with the desiderata formulated at the beginning, we wish to pose a fundamental problem of 
a general relevance within a broad domain of quantum metrology.

Problem 3. Provide general necessary and sufficient conditions, depending on an unspecified probe state $\rho_{\theta}$, relevant for saturability of the quantum Cramér-Rao bound (QCRB) in multiparameter quantum metrology on the single-copy level.

Setup. Given a set of $N>1$ parameters $\boldsymbol{\theta}=$ $\left(\theta_{1}, \ldots, \theta_{N}\right)$ to be estimated, one is concerned with the precision of a chosen estimator $\hat{\boldsymbol{\theta}}$. We follow a usual assumption that the estimator is unbiased. The precision is then most often quantified by the covariance matrix $\operatorname{Cov}(\hat{\boldsymbol{\theta}})$. Due to the famous quantum Cramér-Rao bound we have the lower bound [95-104]

$$
\operatorname{Cov}(\hat{\boldsymbol{\theta}}) \geq Q^{-1},
$$

where the quantum Fisher information matrix is defined as $Q_{k m}=\frac{1}{2} \operatorname{Tr}\left[\rho_{\boldsymbol{\theta}}\left(L_{k} L_{m}+L_{m} L_{k}\right)\right]$. The state $\rho_{\boldsymbol{\theta}}$ depends on the parameters $\boldsymbol{\theta}$ to be estimated and can, in general, be mixed. Moreover, $L_{k}$ is the symmetric logarithmic derivative given through the relation $2 \partial_{k} \rho_{\boldsymbol{\theta}}=$ $L_{k} \rho_{\theta}+\rho_{\theta} L_{k}$, where $\partial_{k}$ is the derivative with respect to the parameter $\theta_{k}$. This is not the only possible choice of the formal setting - one can also, for example, consider bounds involving right logarithmic derivative [105] $\partial_{k} \rho_{\theta}=\rho_{\theta} R_{k}$ - however, here we focus only on the setting with the symmetric logarithmic derivative (other settings described below share many similarities when it comes to the posed problem). The matrix inequality (3), as usual, means that $\operatorname{Cov}(\hat{\boldsymbol{\theta}})-Q^{-1}$ is a positive semidefinite matrix. Therefore, this bound becomes saturated if both matrices entering the QCRB are equal.

For a condensed and comprehensive review of the history of the problem we refer to the "Zeitgeist" section of Ref. [101], where important names such as Helstrom, Belavkin, and Holevo, among others, enter the scene in an appropriate chronological order.

The proposed problem concerns general conditions for saturability of QCRB. By the word "general" we understand that neither the number $N$ of the parameters (and their other features) nor the dimension and the form of the probe state are fixed. Consequently, the necessary and sufficient conditions for saturation of the bound must involve the analyzed mixed state depending on $N$ parameters, $\rho=\rho\left(\theta_{1}, \ldots, \theta_{N}\right)$, and the set of symmetric logarithmic derivatives.

However, there is one crucial distinction we need to make. We assume that only a single copy of the state $\rho_{\theta}$ is available. By allowing $M$ copies of the probe state $\rho_{\theta}^{\otimes M}$ and collective measurements (see Fig. 3 for comparison), we facilitate the asymptotic limit $M \rightarrow \infty$, in which
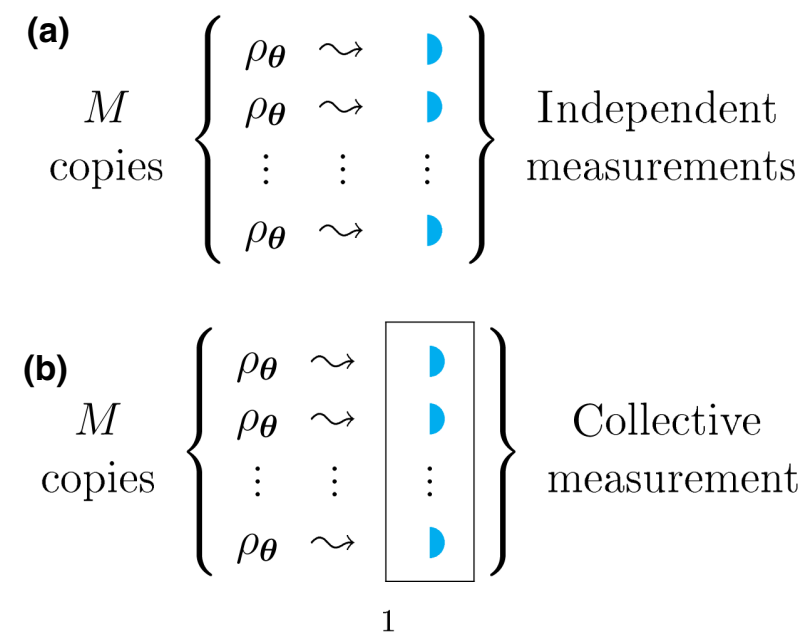

FIG. 3. Pictorial comparison between quantum metrology on the single-copy level (a) and with multiple copies being subject to collective measurements visualized by a frame (b).

the problem has recently been solved [106]. The necessary and sufficient condition in the asymptotic limit (see below) is milder in comparison with the single-copy case (even though here we know only some necessary conditions), because the presence of many copies to some extent sweeps away quantum incompatibility between noncommuting observables.

Below, we provide three particular results as a potential starting point. We stress that we assume $N>1$, as for the case of single-parameter estimation all the conditions mentioned are trivially satisfied. It is well known that QCRB can be saturated if all logarithmic derivatives commute, i.e., $\left[L_{k}, L_{m}\right]=0$, for all $k, m=1, \ldots, N$. This strong condition imposed on the logarithmic derivatives is therefore sufficient. In a special case, when the state $\rho_{\theta}$ is pure, a weak variant of commutativity, i.e., $\operatorname{Tr}\left(\rho_{\theta}\left[L_{k}, L_{m}\right]\right)=0$, for all $k, m=1, \ldots, N$ is proved to be both necessary and sufficient [107]. The latter example, however, does not meet the requirement of generality as assumptions about the probe state appear. On the other hand, the same weak commutativity condition is necessary and sufficient for all states (not necessarily pure), in the aforementioned asymptotic limit [106]. However, on the single-copy level it is known that the situation is more complicated, as yet another necessary condition, situating itself somewhere in between the other conditions, has very recently been established [108]: all logarithmic derivatives need to commute on the whole support of $\rho_{\theta}$ (see Theorem 3 in Ref. [108]).

An assumption which is often made and at the same time jeopardizes generality of the problem is that the parameters to be estimated are imprinted in the probe state by virtue of a unitary transformation, i.e., $\rho_{\theta}=U_{\theta} \varrho U_{\theta}^{\dagger}$. The desired necessary and sufficient conditions shall not depend upon this assumption. 
Looking at the problem from an information-theoretic perspective, the actual mathematical question behind it can be posed in a slightly different way. We are concerned with existence of a POVM (i.e., a measurement) for which the corresponding classical Fisher information matrix equals its quantum counterpart. Given such a measurement one can then resort to a universal construction of a locally unbiased estimator saturating the classical Cramér-Rao bound [see Eq. (9) in Ref. [100] ].

Motivation. Theoretical and experimental quantum information entered the era, when more and more often people speak about quantum technologies. However, with an advanced technology, we usually associate a mindset in which details are tailored to perfection. At the same time, used schemes are being optimized under realistic (not idealized) assumptions.

While the above by itself justifies efforts aiming at closing fundamental gaps in the theoretical framework, there is more to that than just formally oriented curiosity. An increasing effort is and will be put towards "tighter bounds that can be realized for practical applications of multiparameter estimation theory" [109]. It is clear, that such bounds [110], optimization algorithms behind them [109], and experimentally friendly measurement prescriptions [111], will all be accompanied by a serious piece of quantum-theory-oriented mathematics. Therefore, fresh attempts to solve this quite hard and very well-known problem, will render novel techniques and expand the palette of theoretical tools.

Importantly, in multiparameter problems it is in general impossible to obtain saturable matrix inequalities like Eq. (3). One then optimizes scalar figures of merit involving the covariance matrix. A solution to the problem posed here will therefore also tell when the optimal measurement is independent of the figure of merit being chosen.

\section{A. Further perspectives II}

We observe several interesting questions touched upon recently, which would mutually benefit in relation to research devoted to the posed problem. Among such directions we can distinguish the following: quantumness in multiparameter estimation [112,113], uncertainty tradeoffs [114], nonasymptotic regime [115,116] (which is different than the regime considered here), or indefinite causal order [117].

This is by no means an exhaustive list of ideas pertaining to optimal quantum metrology under realistic experimental conditions or with the help of extraordinary protocols. In addition, quantum Fisher information and QCRB are subject of extensive research in the context of thermodynamics [99], and geometry [118]. The latter dates back to the inception of quantum metrology [95], since the relation between Fisher information and metrics on the space of quantum states are very well known [24]. Still, we observe new geometric developments associated with the QCRB, e.g., that in certain cases the bound is independent of the parametrization used in the encoding procedure [118].

Moreover, in Fig. 3 we can only see so-called classicalclassical (a) and classical-quantum (b) settings, since the state $\rho_{\theta}$ is assumed to be given. On the other hand, one can also consider quantum-classical and quantum-quantum strategies (see Fig. 1 in Ref. [96]), relevant when the model $\rho_{\theta}$ is not fixed, or even adaptive strategies (see Fig. 1 in Ref. [119]).

In the context of saturability or attainability (CramérRao, Holevo, etc.) the topic has just been touched upon [120], therefore, we expect more research in this subarea will blossom. Last but not least, even though the Holevo Cramér-Rao bound, a very profound approach, which however is not in the scope of this brief review, has been well understood [121], there are still open problems beyond this paradigm. For example, existence of tight bounds when one cannot perform collective measurements on multiple copies [122].

\section{QUANTUM ENTANGLEMENT AND ITS DISTILLABILITY}

One of the most fundamental notions in the theory of quantum-information processing is that of entanglement. We say that a bipartite product state is called separable with respect to a given bipartition of the system into two distinct physical subsystems (denoted by the tensor product), while all other pure states are entangled. A density matrix representing a mixed state is called entangled if it cannot be represented as a convex combination of product states [123].

Entanglement proved itself to be a crucial resource relevant for quantum-information processing. Therefore, one of the major problems in this field has been, since the early days of quantum information, to decide whether a given quantum state of a composite system is separable or entangled [124].

Perhaps surprisingly, this general problem is to date solved only for $2 \otimes 2$ and $2 \otimes 3$ systems [125], as in these cases the single positive partial transpose criterion provides a constructive answer [126]. Already for a $3 \otimes 3$ system, neither a finite number of positive-maps-based separability criteria [127] nor a technique using finitesize semidefinite programming [128] allows us to conclude whether a given quantum state is entangled or not. Moreover, the known procedure for deciding the separability of a given bipartite quantum state in a finite number of steps [129] cannot be applied in practice due to its high complexity.

The above problem, as well as the whole associated subfield concerned with certification (tests) or quantification (measures) of entanglement, gains visibly less attention in recent years. This occurs likely because a lot has in fact 
been achieved, and it is relatively hard to identify research directions promising an intellectual reward. Not to dream about giving a twist to the whole subfield. Here we attempt to offer two such problems, which, when solved, are capable of boosting the research on quantum entanglement perse.

\section{A. Bound entanglement}

Problem 4. Establish whether there exist bound entangled states with negative partial transpose.

Setup. To analyze quantum entanglement it is useful to introduce the notion of partial transpose of a density matrix. Let $\rho$ denote a bipartite quantum state with matrix elements written in a product basis, $\rho_{i j, l m}=\langle i j|\rho| l m\rangle$. Then its partial transposition, $\rho^{\Gamma}$, reads

$$
\left\langle i j\left|\rho^{\Gamma}\right| \operatorname{lm}\right\rangle=\langle i m|\rho| l j\rangle .
$$

A quantum state is said to have positive partial transpose (PPT) if all eigenvalues of its partial transpose are nonnegative. Otherwise, if some eigenvalues of $\rho^{\Gamma}$ are strictly smaller than zero, the state has negative partial transpose (NPT).

The concept of entanglement distillation refers to protocols that allow us to transform noisy entangled states to maximally entangled states in a well-defined scenario.

To explain the main idea of the concept let us recall that a quantum operation on bipartite quantum system is called of local-operation classical communication (LOCC)-type or just LOCC if and only if it is composed from (possibly quantum) LOs on subsystems and CC (see Ref. [130], Sec. 12.2). Now, the entanglement distillation protocol in its original version is the protocol in which a large number $N$ of copies of $\rho_{A B}$ shared by Alice and Bob can be transformed with the help of a LOCC map into some smaller but still large (e.g., with asymptotically linear scaling with respect to the number of initial states) number $K_{N}$ of maximally entangled two-qubit states, up to some error that vanishes with increasing number of initial pairs.

Typically, the final number of maximally entangled qubit states is replaced by a single maximally entangled state with local dimension $d_{N}=2^{K_{N}}$, which simplifies the description of the whole procedure. For the formal description of the distillation protocol one can see Ref. [130] (Df. 12.1 in Sec. 12.2) and references therein. Now, a given state $\rho_{A B}$ is distillable if and only if it admits entanglement distillation described above. Apart from the explicit construction of the distillation protocol (for specific examples see Ref. [131]), there exist necessary and sufficient tests for that (see Theorem 12.1 of Ref. [130]). One of the crucial and elementary observations is that separable states are not distillable since the tensor product of separable states is still separable, and it is not possible to transform any separable state into an entangled one by LOCC operations.

Entanglement distillation was originally discovered in 1996 when an explicit protocol was proposed for a class of mixed two-qubit states [132] and applied in its general form to quantum error correction [133] and cryptography [134]. Soon after it was shown that all two-qubit entangled states are distillable [135]. Also, in higherdimensional systems most entangled states can be distilled to a singlet form [136], but there exist also nondistillable entangled states, which are called bound entangled $[130,137]$. More specifically, if the dimension of a bipartite system is larger than six, there exist entangled states with PPT property [138] and the set of these states has a positive measure [139]. It has been shown that all entangled states with PPT property are nondistillable [137]. This is the case because LOCC operations used in the entanglement distillation protocol always preserve PPT property, while the desired output states of the distillation protocol - maximally entangled ones - are located outside of the set of PPT states (actually they are far from it with respect to any smooth distance). This fact directly proves that in all dimensions $d d^{\prime} \geq 6$ there exist bound entangled states.

We analyze here Problem 4 with equal dimensions of both subsystems, as the general case, $d \neq d^{\prime}$, can be reduced to it. It has been shown (see Ref. [137]) that a $d$-dimensional [140] bipartite state $\rho$ defined on a composite Hilbert space $\mathcal{H}_{d} \otimes \mathcal{H}_{d}$ is distillable, if it is $n$-copy distillable for some finite $n$. The latter notion is of a rather technical character, being related with the original definition of distillability, but at the same time having a quite different mathematical sense. Namely, the property of $n$-copy distillability means that there exist twodimensional (i.e., of rank-two) projectors $P$ and $Q$ such that the matrix $(P \otimes Q)\left[\rho^{\Gamma}\right]^{\otimes n}(P \otimes Q)$ has a negative eigenvalue $[130,137,141]$. It should be stressed that the projectors $P$ and $Q$ act on the product $\left(\mathcal{H}_{d}\right)^{\otimes n}$ of all $n$ Hilbert spaces associated with left and right subsystems of copies of the considered bipartite system, respectively — see Refs. $[142,143]$.

The question of NPT bound entanglement is closely related to a mathematical problem concerning 2-copositive maps $[142,144]$. A linear map $\Lambda: M_{d}(\mathcal{C}) \rightarrow M_{d}(\mathcal{C})$ acting on $\mathcal{H}_{d}$ is called positive if and only if it transforms any matrix with non-negative eigenvalues into a matrix with the same property. Furthermore, a linear map $\Lambda$ is called $k$-positive if and only if the following extension $\mathbb{1}_{k} \otimes \Lambda: M_{k}(\mathcal{C}) \otimes M_{d}(\mathcal{C}) \rightarrow M_{k}(\mathcal{C}) \otimes M_{d}(\mathcal{C})$ is positive, where $\mathbb{1}_{k}$ stands for the identity map, which sends any complex matrix from $M_{k}(\mathcal{C})$ into itself. In particular, 1positivity is equivalent to positivity. The map is called completely positive if and only if it is $k$-positive for any $k$. For a finite dimension $d$, to ensure complete positivity it is enough to check only $k$-positivity for $k=d$. A map $\Lambda$ 


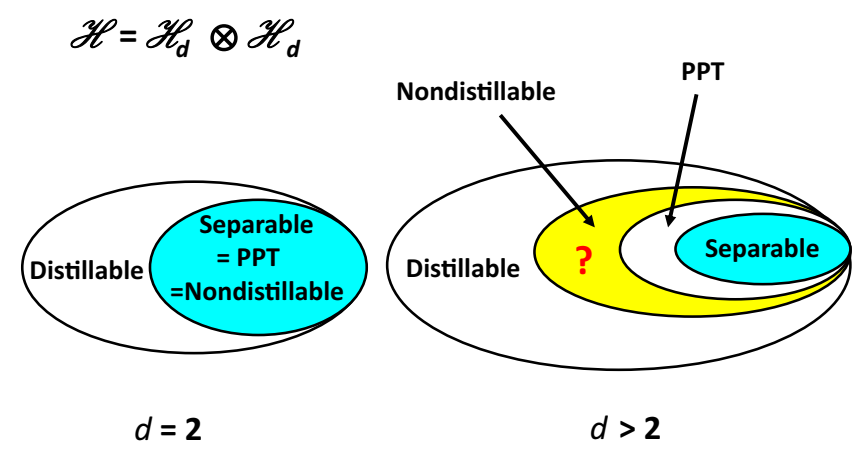

FIG. 4. In the two-qubit problem, $d=2$, the set of separable states coincides with the set of PPT states and there are no bound entangled states since any entangled state is distillable. For higher dimensions, $d>2$, one asks whether the hypothetical region representing bound entangled states with negative partial transpose, depicted in yellow, is empty or not.

is called $k$-copositive (respectively, completely co-positive) if and only if the composition $S=T \circ \Lambda$ is $k$ positive (respectively, completely positive), where $T$ stands for transposition. In particular, 1-copositive maps are called just co-positive.

Motivation. This is one of the long-standing open questions of quantum-information theory [142,145]. It provides a sharp distinction between the two-qubit case, in which all entangled states are distillable [135], and higherdimensional $d \otimes d$ problem, for which the question of existence of bound entangled states with negative partial transpose is open - see Fig. 4.

Its positive solution would therefore have several consequences. If NPT bound entangled states exist then the set of nondistillable entangled states is neither closed under the tensor product nor under mixing (see Ref. [144], Sec. 4.4 , for a detailed proof). The latter means that there would exist a pair of nondistillable entangled states such that their tensor product — or mixture, respectively — were distillable. This would imply that one of the central measures of entanglement theory, namely distillable entanglement (which describes an asymptotic amount of entanglement that can be distilled from many copies of a given state by local operations and classical communication [124]) is neither additive nor convex [144].

In this way, a possible affirmative solution to the present problem would lead to an extremal example of superadditivity. Namely, it has been proven that for any NPT state there exists PPT bound entangled state such that the product of the two is distillable [146]. Consequently, if the NPT state were bound entangled, then we would have the pair of two bound entangled states (both with their individual distillable entanglement measure equal to zero) such that their tensor product would be distillable (i.e., having the measure strictly positive). As already pointed out, such a scenario is an extreme case of superadditivity: two objects containing no resource of a given type, if put together constitute a single object that, surprisingly, turns out to contain some amount of the resource. For this type of effect on the ground of quantum channel capacities see Ref. [147].

One can show [142] that the existence of an $n$-copy nondistillable state is equivalent to the existence of a completely positive map $\Lambda$ such that it is not completely copositive but 2-copositive and its $n$th tensor power, $\Lambda^{\otimes n}=\Lambda \otimes$ $\cdots \otimes \Lambda$, is also 2-copositive. Interestingly, there is also sufficient condition for existence of NPT bound entanglement expressed in the language of positive maps. If there exists a positive map $\Lambda$ that is neither completely positive, nor completely copositive such that its tensor power $\Lambda^{\otimes n}$ is positive for any $n$, (this property is called tensorstable positivity), then there exist NPT bound entangled states that can be constructed explicitly on the basis of this map [148].

Note that for any $n$ there exists an $n$-copy nondistillable state that is $(n+1)$-copy distillable - see Ref. [149]. This fact might be considered as an indication that the present problem of existence of NPT bound entanglement is hard.

An important practical observation bridging between the fourth and the fifth problem is contained in the following theorem [136]: NPT nondistillable entanglement exists if and only if there exist NPT nondistillable entangled Werner states for local dimension $d>2$. In the smallest dimension, $d=2$, this is not the case.

This theorem implies that the current, fourth problem can be formulated as follows: decide whether there exists a NPT Werner state, which is nondistillable.

Werner states [123] constitute a one-parameter family $\rho(d, \alpha)$ of density matrices of order $d^{2}$ described in the next section. These states are NPT for $\alpha \in[-1,-1 / d)$ and they are 1-copy nondistillable for $\alpha \in[-2 / d, 1]$. In this range of the parameter $\alpha$ they are conjectured to be nondistillable [142-145,150].

Although the above theorem reduces the problem of NPT bound entangled states to the question concerning a single-parameter family, it is not clear, whether analysis of this particular family of states provides the easiest technical way to solve the problem. Some other subfamilies of the set of NPT states were also considered in the above context $[142,149]$. Finally, it is worth mentioning that a variant of the Problem 4, for quantum states defined with help of hypercomplex numbers, has recently been stated and solved affirmatively. However, this solution cannot be extended to standard quantum description in a natural way [151].

\section{B. Distillability of quantum entanglement}

Problem 5. Decide whether the Werner state $\rho(4,-1 / 2)$ of two ququarts, $d=4$, defined in Eq. (5) below, is 2-copy distillable. 


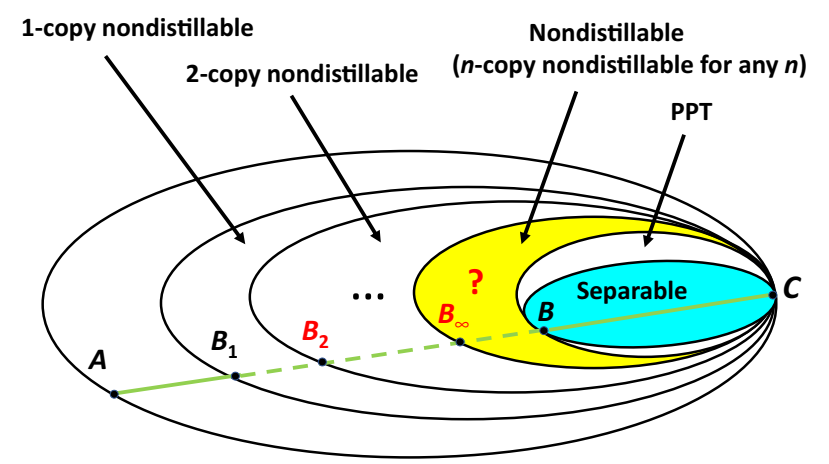

FIG. 5. Sketch of the convex set of mixed quantum states for a $d \otimes d$ system with $d>2$, which contains the sets of separable states, a larger set of PPT states and the sets of states with various classes of distillability. The line represents the family of Werner states, Eq. (5), and the points $A, B_{1}, B$, and $C$ correspond to states labeled by $\alpha$ equal to $-1,-2 / d,-1 / d$, and 1, respectively. Point $A$ represents here the mixed state equal to the normalized projector onto the antysymmetric subspace. Problem 4 of the existence of NPT bound entanglement is equivalent to the question, whether point $B_{\infty}$, the position of which is still unknown, differs from point $B$. The nature of the states along the dashed line $B_{1} B$ is still unclear - to solve Problem 5 one has to decide, whether in case $d=4$ the unknown point $B_{2}$ is identical with $B_{1}$.

Setup. Consider the family of Werner states defined on the Hilbert space $\mathcal{H}_{d} \otimes \mathcal{H}_{d}$ as

$$
\rho(d, \alpha)=\frac{\mathbb{1} \otimes \mathbb{1}+\alpha V}{d^{2}+\alpha d},
$$

with the general range of the parameter $\alpha \in[-1,1]$. The matrix $V$ stands for the swap operator, defined by its matrix elements, $\langle i j|V| k l\rangle=\delta_{i l} \delta_{j k}$. Let us repeat that the above states are NPT for $\alpha \in[-1,-1 / d)$ and our fourth problem can be just reduced to the analysis of their distillability in the cases of $d>2$. A distinguished state of this family, $\rho(4,-1 / 2)$, appearing in the problem considered here, is the only two-ququart Werner state such that its partial transpose [see Eq. (4)] is proportional to a unitary matrix.

The Werner states are invariant with respect to twirling with local unitaries [123], so they are also called $U \otimes$ $U$ invariant. It was conjectured [142-145,150] that the Werner states, which are not 1-copy distillable, are just nondistillable, so in particular they are also 2-copy nondistillable. Problem 5 is visualized in Fig. 5, and can be reduced to the question whether in the case $d=4$ the points $B_{1}$ and $B_{2}$ in this plot are equal. A stronger conjecture that for Werner states' 1-copy nondistillability is equivalent to complete nondistillability, means that $B_{\infty}=$ $B_{1}$ in Fig. 5.

Motivation. On the physical side working on this problem might bring a step towards a proof of existence of NPT bound entanglement discussed in Problem 4. Note that there is no promise for that due to the examples of Watrous [149], in which the corresponding nondistillability property holds for $n$ copies, but not for $n+1$ copies. Yet, some hope might come from the fact that Watrous states do not have full rank - as opposed to the above Werner states - and this particular property seems important for their distillability.

Furthermore, a possibly negative answer would render the chance for universality of distillation protocols (cf. Refs. [135,136]), involving the recurrence scheme [129] as a first stage of interaction among particles. The recurrence scheme is very intuitive (can be seen as sequence or probabilistic pumping of entanglement from one pair to another) and requires very simple two-particle coupling, namely CNOT gates. On the other side, the positive solution of the problem would allow construction of practical entanglement distillation scheme for remarkably noisy states.

On a mathematical side, the negative solution of the problem would provide a very elegant completely positive map that is not completely co-positive, but it is 2-copositive and the tensor product of its two copies also possesses this property. Interestingly, this question is also equivalent to the following algebraic problem [143]: show that the sum of squares of the two largest singular values is bounded by $1 / 2$ for any Kronecker (tensor) sum, $A \oplus B=A \otimes \mathbb{1}+\mathbb{1} \otimes B$, where $A$ and $B$ denote traceless matrices of size 4 satisfying $\operatorname{Tr}\left(A^{\dagger} A\right)+\operatorname{Tr}\left(B^{\dagger} B\right)=1 / 4$. The bound equal to $1 / 2$ has been proven [143] under the additional assumption that $A$ and $B$ are normal, so they commute with their Hermitian conjugates. Recently, further progress has been announced in the form of a theorem stating that the bound still holds if one of the matrices is made completely arbitrary [152].

Moreover, the explicit parameters $d=4$ and $\alpha=-1 / 2$ appearing in the problem are of special interest since they correspond to the case of what follows:

(i) The minimal dimension for which this very special Werner state is 1-copy nondistillable. Namely, the state $\rho(d,-2 / d)$ has its partial transpose proportional to the dichotomic unitary operator $U=$ $I-2\left|\psi_{+}\right\rangle\left\langle\psi_{+}\right|$, where $\left|\psi_{+}\right\rangle$denotes the maximally entangled state defined in Eq. (A1). A dichotomic unitary operator by definition has eigenvalues \pm 1 .

(ii) The unique dimension, for which the above Werner state characterized by the parameter $\alpha=-2 / d$ is located just on the boundary of a 1-copy nondistillability. For $d=4$ all the states with $\alpha<-1 / 2$ are already one-copy distillable, which is not true for $d>4$.

The choice of the state with its partial transpose proportional to the dichotomic unitary operator is additionally motivated by the fact that checking its $n$-copy distillability 
seems to be easier than in the general case. In particular, the property of proportionality to the dichotomic unitary operation is preserved with respect to taking the tensor product.

\section{Further perspectives III}

Solution of the fifth problem would likely provide us some new insight into general properties of the Kronecker sum of matrices, $A \oplus B=A \otimes \mathbb{1}+\mathbb{1} \otimes B$, relevant from the point of view of quantum theory and already studied in general matrix analysis [153]. Problem 4 seems to be rather complex, so its solution may involve some novel techniques concerning tensor products of several matrices. Its negative solution would likely stimulate research on an important question relevant for quantum communication, namely, whether all NPT entangled states represent quantum privacy that may be distilled to the so-called private bit states - see Ref. [154]. If the answer were positive, the practical question would be, whether such a distillation procedure can be achieved in the scheme involving socalled "one-way classical communication," in which one party, say Alice, communicates classical bits to Bob, and not vice versa.

\section{CONCLUDING REMARKS}

The goal of this perspective and the competition announced is to stimulate further research on interesting mathematical problems directly related to quantuminformation applications. Each problem described above has in a way been associated to a single simple equation, which played a profound role in the development of the theory of quantum information. Furthermore, each problem is illustrated with a single figure, aimed to visualize the question posed.

The problems concerning the discrete Hilbert space, related to deep algebraic and geometric properties of the set of quantum states, are also linked to fundamental problems from various branches of mathematics ranging from group theory to number theory. Solving some of them will impact the research on existence and enumeration of various constellations of quantum states, which satisfy certain conditions of balance and symmetry - a novel emerging field at the borderline of theoretical physics and mathematics, which can be called "quantum combinatorics."

Results in the outlined directions, including metrology and the QCRB, while relevant for our understanding of foundations of quantum theory, can be useful for the development of quantum-information processing. Furthermore, some measurement schemes or particular constellations of quantum states can influence the computer designed quantum experiments [155], which may allow one to cope with a huge number of possible configurations, which "explodes combinatorically." On the other hand, it is also thinkable that the future results of new physical experiments designed in this way could bring hints concerning some of the theoretical problems discussed in this work.

Possible solutions of problems devoted to distillability of quantum entanglement definitely would enrich our understanding of the nature of quantum interaction as a resource in entanglement processing - one of the key ingredients of quantum advantage in information processing. This knowledge might be relevant for development of secure quantum communication in quantum networks. For instance, NPT bound entanglement can lead to private bits that will allow to go beyond limitations known in quantum repeaters for PPT states with quantum security - see Ref. [156]. Independently, the solution will shed new light on important structures known in linear matrix algebra.

\section{ACKNOWLEDGMENTS}

We are pleased to thank Francesco Albarelli, Ingemar Bengtsson, Adam Burhardt, Rafał DemkowiczDobrzański, Dardo Goyeneche, Markus Grassl, Otfried Gühne, Michał Horodecki, Felix Huber, Jędrzej Kaniewski, Arul Lakshimnarayan, Marcin Marciniak, Albert Rico, Wojciech Słomczyński, and Oliver Reardon-Smith for numerous helpful remarks and suggestions, and to Jakub Czartowski for preparing two figures. We also acknowledge Bogdan Cichocki who has been introducing The Bald Soprano, in the context used in Sec. II, to many generations of physics students at the University of Warsaw. P.H. and Ł.R. would like to acknowledge support by the Foundation for Polish Science (IRAP project, ICTQT, Contract No. 2018/MAB/5, cofinanced by the EU within

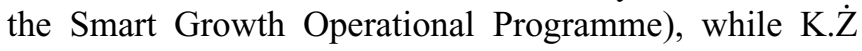
acknowledges support by Narodowe Centrum Nauki under the Maestro Grant No. DEC-2015/18/A/ST2/00274 and by the Foundation for Polish Science under the project Team-Net NTQC No. 17C1/18-00.

\section{APPENDIX A: QUANTUM ORTHOGONAL LATIN SQUARES}

Problem 6. [158] Determine whether there exists a pair of quantum orthogonal Latin squares [159-161] of order six. In other words, find a solution of the problem of 36 "entangled officers" of Euler or demonstrate that it does not exist.

Setup. A Latin square of order $N$ is filled with $N$ copies of $N$ symbols arranged in a square in such a way that no row nor column of the square contains the same symbol twice. The name refers to papers of Leonhard Euler [162], who used Latin characters as symbols to be arranged. To enjoy a simple example use the Pauli matrix and write down $2 \sigma_{x}+\mathbb{1}_{2}$. It is likely that Euler's approach to the problem was rather different. Two orthogonal Latin 


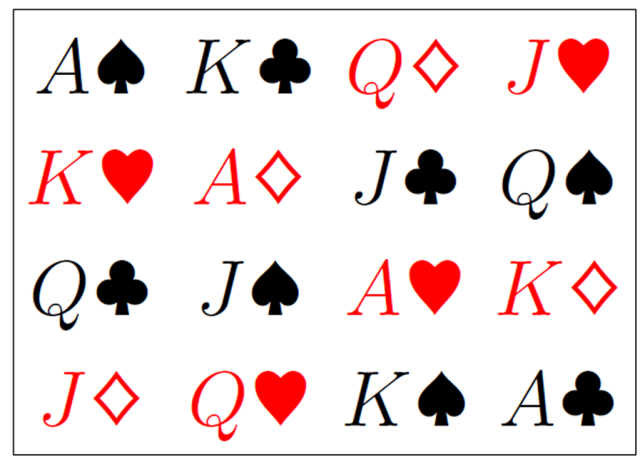

FIG. 6. Example of $N=4$ Greaco-Latin square prepared for bridge players. Due to works of Euler and Tarry we know that for $N=6$ a similar design of 36 cards of six different suits and six different ranks (or 36 officers of different ranks and arms) does not exist. Is there a solution of the $N=6$ problem if we play bridge with quantum cards, like $(|K \boldsymbol{\$}\rangle+|Q \diamond\rangle) / \sqrt{2}$, or allow the officers of Euler to be entangled?

squares (also called Graeco-Latin squares) of order $N$ consist of $N^{2}$ cells arranged in a square with a pair of ordered symbols in each cell, for instance, one Greek character and one Latin. Every row and every column of the square contains each possible pair of symbols exactly once, and no two cells contain the same ordered pair — see Fig. 6 prepared for bridge players [163]. A set of $k$ Latin squares, which are pairwise orthogonal, are called mutually orthogonal Latin squares (MOLSs). It is easy to show that for a given $N$ there exist no more than $N-1$ MOLSs. Similarly with the case of the MUBs, this bound is saturated if $N$ is a prime or a power of a prime [164].

Historically, Euler analyzed the problem of 36 officers from six regiments, each containing six officers of six different ranks. They should be arranged before a parade into a $6 \times 6$ square such that each row and each column holds only one officer from each regiment and only one officer from each rank. Euler wrote in 1782 that this problem has no solution [162] without providing a formal proof, established only in 1901 by Gaston Tarry [165]. This result implies that there is no pair of orthogonal Latin squares of size 6, so that the upper bound for the number of MOLS, in this case $N-1=5$, is not saturated. For any $N \geq 7$ there exist at least two MOLSs, in particular, also for $N=2 \times 5=10$ - consult a novel by Georges Perec [166]. In general, the problem of finding the maximal number of MOLSs for an arbitrary value of $N$ remains open [167].

As a rule of thumb, for any interesting classical notion one can find a quantum analog. A quantum Latin square is an $N \times N$ table of $N^{2}$ vectors from $N$-dimensional Hilbert space $\mathcal{H}_{N}$ arranged in such a way that every row and every column of the table forms an orthonormal basis in the space [168].

Orthogonal quantum Latin squares (OQLSs).
To discuss a quantum analog of two orthogonal Latin squares consider a collection of $N^{2}$ normalized vectors $\left|\psi_{i j}\right\rangle$ from a composite space, $\mathcal{H}_{N} \otimes \mathcal{H}_{N}$ [159-161]. Any such state can be written in a product basis, $\left|\psi_{i j}\right\rangle=$ $\sum_{k, \ell=1}^{d} X_{k \ell}^{i, j}|k, \ell\rangle$ for $i, j=1, \ldots, d$.

A set of $N^{2}$ bipartite states $\left|\psi_{i j}\right\rangle$ forms a OQLS if

(a) the states satisfy ortogonality relations, $\left\langle\psi_{i j} \mid \psi_{k \ell}\right\rangle=$ $\delta_{i k} \delta_{j \ell}$, so they form an orthonormal basis, and the block matrix $\tilde{X}$ of size $N^{2}$, written $\tilde{X}=$ $\left(X^{1,1}, \ldots, X^{1, N} ; \ldots ; X^{N, 1}, \ldots, X^{N, N}\right)$, is block unitary, so that the conditions

(b) $\sum_{i=1}^{N} X^{i, j}\left(X^{i, \ell}\right)^{\dagger}=\delta_{j, \ell} \mathbb{I}$, and

(c) $\sum_{j=1}^{N} X^{i, j}\left(X^{k, j}\right)^{\dagger}=\delta_{i, k} \mathbb{I}$, are satisfied.

Any Graeco-Latin square leads to such a design, since it suffices to treat the pair of classical objects $(\alpha, B)$ as a product state, $|\alpha\rangle \otimes|B\rangle[19]$.

It is known that the generalized Bell state

$$
\left|\psi_{+}\right\rangle=\frac{1}{\sqrt{N}} \sum_{j=1}^{N}|j\rangle \otimes|j\rangle,
$$

is maximally entangled among all states of a bipartite $N \times N$ system. For other systems, a natural question arises [169-178]: what are the most entangled states for quantum systems consisting of $N$ systems with $d$ levels each? The answer depends on the entanglement measure used [24], but already for a four-qutrit system there exists a state, which displays maximal entanglement with respect to all three possible splittings of the entire system into two pairs of qutrits. Such a state is called maximally multipartite entangled state (MMES) [175] or absolutely maximally entangled (AME) state [179].

This notion can also be generalized for larger systems. An $n$-partite pure state is called AME state if it is maximally entangled with respect to all possible bipartitions [179], so that all its reductions consisting of $k$ subsystems, with arbitrary $k \leq\lfloor n / 2\rfloor$, are maximally mixed. A density matrix on a given $N$-dimensional Hilbert space is maximally mixed, if it is proportional to the identity operator on this space, $\rho_{*}=\mathbb{1}_{N} / N$. It is known that there are no AME states of four qubits [170], and equivalently, a pair of OQLSs does not exist for $N=2$. This problem was found interesting to a future Nobel laureate, who showed with his collaborators a relation between this fact and frustration in the spin systems [175].

Scott demonstrated [172] that, for systems containing $m$ qubits, the AME states do exist for $m=3,4,5,6$ and also showed that they do not exist for $m \geq 8$. The last remaining issue of $m=7$ qubits was later solved by Huber et al. [180], who proved that such AME states do not exist. The list of currently known AME states is available online [181]. 
As there are no two orthogonal Latin squares (OLSs) of order six, the famous classical problem of 36 officers of Euler has no solution [182].

An analogous quantum problem, which involves 36 entangled officers, was open until very recently [19]

Motivation. This problem can be reformulated in several other settings. To present them we need to review some further notions. A unitary matrix $U$ of size $N^{2}$ is called 2-unitary [183] if both the partially transposed matrix $U^{\Gamma}$ and the reshuffled matrix $U^{R}$ remain unitary - for the definitions of these reorderings of the entries of a matrix see Eq. (4) and consult Ref. [24].

Any matrix of a square size can be represented as a tensor $T_{i j k l}$ with four indices. Such a tensor can be reshaped into a matrix $W_{\mu \nu}$ using composed indices in three different ways: (a) $\mu=\mu(i j)$, (b) $\mu=\mu(i k)$, and (c) $\mu=\mu(i l)$, while the second index $v$ is obtained in each case from the remaining two indices. A tensor $T$ is called perfect [184] if for any of these three ways of reshaping it, the outcome matrix $W$ becomes unitary. Hence any flattening of a perfect tensor forms 2-unitary matrix.

Establishing a positive result concerning existence of a pair of quantum orthogonal Latin squares of order six is equivalent to proving that

(i) there exists AME state of four subsystems with six levels each $[179,185]$. Thus there exists the corresponding quantum error-correction code [186], written $[(4,1,3)]_{6}$;

(ii) there is 2-unitary matrix $U \in \mathcal{U}(36)$;

(iii) there exists a perfect tensor with four indices, each running from 1 to 6 .

Furthermore, a positive result can become an important step towards development of the theory of quantum combinatorial designs, which deals with particular constellations of discrete quantum objects, with special properties of symmetry and balance, hidden in the continuous Hilbert space. As the standard combinatorics deals with discrete objects and is related to the group of permutations, its quantum analog concerns the continuous space of quantum states and relies on the continuous unitary group.

Since the problems, number 2 and number 6 , refer to the same dimension, $N=6$, it is natural to speculate that they might be somehow related. It seems, however, that a connection between problems of finding the maximal number of MOLSs and MUBs for a given dimension is not a direct one $[81,82]$. On the other hand, several links between both problems were established: Wocjan and Beth used (classical) MOLSs to construct a set of six MUBs in dimension $N=26^{2}=676$, which beats the prime power construction applied to the factorization $N=2^{2} 13^{2}$ yielding only $4+1=5$ MUBs [54]. Furthermore, Musto used quantum Latin squares to construct in square dimensions mutually unbiased bases consisting of maximally entangled states [187].

\section{Further perspectives IV}

Incidentally, Problem 6 concentrates on the Hilbert space of dimension six, but it is clear that more than a single copy of $\mathcal{H}_{6}$ has to be involved. Looking for a solution of the generalized Euler problem we are allowed to play with quantum cards, $\cos \varphi|A \boldsymbol{\rangle}\rangle+\sin \varphi|K \odot\rangle$, and analyze configurations of $6^{2}=36$ possibly entangled states in $\mathcal{H}_{6} \otimes \mathcal{H}_{6}$

To create entanglement in a bipartite $N \times N$ system we need a global unitary gate, $U \in \mathcal{U}\left(N^{2}\right)$, which couples both subsystems. For any such bipartite gate $U$ one defines an entangling power $[172,182,188]$ as the average linear entropy of entanglement [24] created when $U$ acts on a random product state sampled according to the Haar measure on both subspaces.

A unitary matrix $U$ of size $N^{2}$, which saturates the absolute bound for the entangling power, has to be 2-unitary and therefore it allows one to construct the AME state for four subsystems with $N$-levels each - see Ref. [189]. In the latter formulation of the problem devoted to the search of distinguished AME pure states of four parties $[179,181]$, one works with four subsystems with six levels each, which are represented in the space $\mathcal{H}_{6}^{\otimes 4}$.

To shed some light on counterintuitive properties of such a peculiar quantum state consider a set of four unbiased cube dice in four different colors. Ask your friend to select any two of them and to roll them in the same moment, as you roll the remaining two dice. If the set of these four dice is prepared in a four-quhex AME state, then by measuring the outcomes of both your dice you will always be able to predict the outcomes of both dice rolled by your friend. This is not possible if the four dice are replaced by four coins, as AME states of four qubits do not exist [170].

Interestingly, the existence problem of AME states can be mapped into a question concerning properties of certain mixed states of the squared dimension. It was recently demonstrated [190] that existence of the desired AME state of four subsystems with six levels each is equivalent to the bipartite separability of a certain mixed state $\rho$ living in $\mathcal{H}_{6}^{\otimes 8}=\mathcal{H}_{6}^{\otimes 4} \otimes \mathcal{H}_{6}^{\otimes 4}$

[1] R. S. Ingarden, Quantum information theory, Rep. Math. Phys. 10, 43 (1976).

[2] C. H. Bennett and D. P. DiVincenzo, Quantum information and computation, Nature 404, 247 (2000).

[3] M. A. Nielsen and I. L. Chuang, Quantum Computation and Quantum Information (Cambridge University Press, Cambridge, Cambridge, 2010), 2nd ed.

[4] S. Wiesner, Conjugate coding, SIGACT News 15, 78 (1983).

[5] C. H. Bennett and G. Brassard, in Proceedings of the IEEE International Conference on Computers, Systems, and Signal Processing, Bangalore, 175 (1984). 
[6] A. Ekert, Quantum Cryptography Based on Bell Theorem, Phys. Rev. Lett. 67, 661 (1991).

[7] C. Bennett and S. Wiesner, Communication via Oneand Two-Particle Operators on Einstein-Podolsky-Rosen State, Phys. Rev. Lett. 69, 2881 (1992).

[8] C. H. Bennett, G. Brassard, C. Crepeau, R. Jozsa, A. Peres, and W. K. Wootters, Teleporting an Unknown Quantum State via Dual Classical and Einstein-Podolsky-Rosen Channels, Phys. Rev. Lett. 70, 1895 (1993).

[9] B. Schumacher, Quantum coding, Phys. Rev. A 51, 2738 (1995).

[10] D. Deutsch and R. Jozsa, Rapid solutions of problems by quantum computation, Proc. Royal Soc. Lond. A 439, 553 (1992).

[11] P. W. Shor, in Proc. 35th Annual Symposium on the Foundations of Computer Science, 124 (IEEE Computer Society Press, Los Alamitos, California, 1994).

[12] L. K. Grover, Quantum Mechanics Helps in Searching for a Needle in a Haystack, Phys. Rev. Lett. 79, 325 (1997).

[13] M. Gessner, C. Fabre, and N. Treps, Superresolution Limits from Measurement Crosstalk, Phys. Rev. Lett. 125, 100501 (2020).

[14] M. Tsang, R. Nair, and X. M. Lu, Quantum Theory of Superresolution for Two Incoherent Optical Point Sources, Phys. Rev. X 6, 031033 (2016).

[15] For instance, within the European Quantum Flagship initiative, quantum technologies are split into the following five pillars: quantum communication, quantum simulations, quantum sensing and metrology, quantum computing, quantum basic science.

[16] Finding a correct answer to any of the five problems presented above will be rewarded by the Golden KCIK Award5 established by the National Quantum Information Centre (KCIK) in Poland. Each year up to two prizes can be awarded (see the KCIK webpage 8, https://kcik.ug.edu.pl/post.php?id=1981). The competition will be closed if all five problems are solved. Then, as always happens, new problems will come into play.

[17] While presenting the problems we used notation common in each subfield. Thus the reader is advised that the notation is not entirely consistent throughout the entire paper. Consequently, in different problems the symbols $n, d, k$, and $N$ have different meaning.

[18] E. Ionesco, The Bald Soprano, and Other Plays (Grove Press, New York, New York, 1982).

[19] S. Ahmad Rather, A. Burchardt, W. Bruzda, G. RajchelMieldzioć, A. Lakshminarayan, and K. Życzkowski, Thirty-six Entangled Officers of Euler: Quantum Solution to a Classically Impossible Problem, Phys. Rev. Lett. 128, 080507 (2022).

[20] G. Zauner, Quantendesigns - Grundzüge einer nichtkommutativen Designtheorie. Dissertation, Universität Wien, 1999; English translation, Int. J. Quantum Inf. 9, 445 (2004).

[21] J. M. Renes, R. Blume-Kohout, A. J. Scott, and C. M. Caves, Symmetric informationally complete quantum measurements, J. Math. Phys. 45, 2171 (2004).

[22] D. Andersson, An Enthusiast's Guide to SICs in Low Dimensions, Master Thesis, Stockholm University, (2015), http://www.diva-portal.org/smash/get/diva2: 1316066/FULLTEXT01.pdf.
[23] B. C. Stacey, Maximal sets of equiangular lines, ArXiv:2008.13288.

[24] I. Bengtsson and K. Życzkowski, Geometry of Quantum States. An Introduction to Quantum Entanglement, II edition, Cambridge University Press, Cambridge 2017.

[25] A. J. Scott and M. Grassl, SIC-POVMs: A new computer study, J. Math. Phys. 51, 042203 (2010).

[26] A. J. Scott, SICs: Extending the list of solutions, preprint ArXiv:1703.03993.

[27] C. A. Fuchs, M. C. Hoang, and B. C. Stacey, The SIC question: History and state of play, Axioms 6, 21 (2017).

[28] M. Grassl, Finding Numerical and Exact Solutions to the SIC-POVM Problem, talk at the Max Planck Institute for the Science of Light, Erlangen, Division F. Marquardt, 03.11.2020 - see also http://sicpovm.markus-grassl.de.

[29] M. Appleby, T-Y. Chien, S. Flammia, and S. Waldron, Constructing exact symmetric informationally complete measurements from numerical solutions, J. Phys. A 51, 165302 (2018).

[30] M. Grassl and A. J. Scott, Fibonacci-Lucas SIC-POVMs, J. Math. Phys. 58, 122201 (2017).

[31] M. Appleby and I. Bengtsson, Simplified exact SICs, J. Math. Phys. 60, 062203 (2019).

[32] M. Appleby, I. Bengtsson, M. Grassl, M. Harrison, and G. McConnell, SIC-POVMs from Stark Units, (2021), preprint ArXiv:2112.05552.

[33] H. Zhu, SIC-POVMs and Clifford groups in prime dimensions, J. Phys. A 43, 305305 (2010).

[34] J. Jedwab and A. Wiebe, A simple construction of complex equiangular lines, in Algebraic Design Theory and Hadamard Matrices. ed. C. Colbourn, Springer, Cham (2015).

[35] M. Appleby, I. Bengtsson, I. Dumitru, and S. Flammia, Dimension towers of SICs. I. Aligned SICs and embedded tight frames, J. Math. Phys. 58, 112201 (2017).

[36] O. Andersson and I. Dumitru, Aligned SICs and embedded tight frames in even dimensions, J. Phys. A 52, 425302 (2019).

[37] I. Bengtsson, Algebraic units, anti-unitary symmetries, and a small catalogue of SICs, Quantum Inf. Comput. 20, 0400 (2020).

[38] D. M. Appleby, H. Yadsan-Appleby, and G. Zauner, Galois automorphisms of a symmetric measurement, Quantum Inf. Comput. 13, 672 (2013).

[39] M. Appleby, S. Flammia, G. McConnell, and J. Yard, SICs and algebraic number theory, Found. Phys. 47, 1042 (2017).

[40] G. S. Kopp, SIC-POVMs and the Stark conjectures, Int. Math. Res. Not. 2021, 13812 (2021).

[41] I. Bengtsson, SICs: Some explanations, Found. Phys. 50, 1794 (2020).

[42] I. D. Ivanović, Geometrical description of quantal state determination, J. Phys. A 14, 3241 (1981).

[43] W. K. Wootters and B. D. Fields, Optimal statedetermination by mutually unbiased measurements, Ann. Phys. 191, 363 (1989).

[44] A. Klappenecker and M. Rötteler, Construction of MUBs, Lect. Notes Comp. Sci. 2948, 137 (2004).

[45] M. Weiner, A gap for the maximum number of mutually unbiased bases, Proc. Amer. Math. Soc. 141, 1963 (2013). 
[46] S. Bandyopadhyay, P. O. Boykin, V. Roychowdhury, and F. Vatan, A new proof for the existence of MUBs, Algorithmica 34, 512 (2002).

[47] C. Archer, There is no generalization of known formulas for MUBs, J. Math. Phys. 46, 022106 (2005).

[48] S. Brierley, S. Weigert, and I. Bengtsson, All mutually unbiased bases in dimensions two to five, Quantum Info. Comp. 10, 0803 (2010).

[49] J. Lawrence, Mutually unbiased bases and trinary operator sets for $N$ qutrits, Phys. Rev. A 70, 012302 (2004).

[50] J. L. Romero, G. Björk, A. B. Klimov, and L. L. SánchezSoto, Structure of the sets of mutually unbiased bases for $N$ qubits, Phys. Rev. A 72, 062310 (2005).

[51] M. Combescure, Block-circulant matrices with circulant blocks, Weil sums, and mutually unbiased bases. II. The prime power case, J. Math. Phys. 50, 032104 (2009).

[52] M. Wieśniak, T. Paterek, and A. Zeilinger, Entanglement in mutually unbiased bases, New J. Phys. 13, 053047 (2011).

[53] D. McNulty, B. Pammer, and S. Weigert, Mutually unbiased product bases for multiple qudits, J. Math. Phys. 57, 032202 (2016).

[54] P. Wocjan and T. Beth, New construction of MUBs in square dimensions, Quant. Inf. Comput. 5, 181 (2005).

[55] T. Durt, B.-G. Englert, I. Bengtsson, and K. Życzkowski, On mutually unbiased bases, Int. J. Quant. Inf. 8, 535 (2010).

[56] G. Alber and C. Charnes, Mutually unbiased bases: A group and graph theoretical approach, Phys. Scripta 94, 014007 (2018).

[57] I. Bengtsson, W. Bruzda, A. Ericsson, J.-A. Larsson, W. Tadej, and K. Życzkowski, MUBs and Hadamards of order six, J. Math. Phys. 48, 052106 (2007).

[58] P. Butterley and W. Hall, Numerical evidence for the maximum number of mutually unbiased bases in dimension six, Phys. Lett. A 369, 5 (2007).

[59] S. Brierley and S. Weigert, Constructing mutually unbiased bases in dimension six, Phys. Rev. A 79, 052316 (2009).

[60] M. Grassl, On SIC-POVMs and MUBs in Dimension 6, preprint ArXiv:quant-ph/0406175 (v2 2009).

[61] P. Jaming, M. Matolcsi, P. Móra, F. Szöllösi, and M. Weiner, A generalized Pauli problem and an inffinite family of MUB-triplets in dimension 6, J. Phys. A 42, 245305 (2009).

[62] P. Jaming, M. Matolcsi, and P. Móra, The problem of mutually unbiased bases in dimension 6, Cryptography Commun. 2, 211 (2010).

[63] P. Raynal, X. Lü, and B.-G. Englert, Mutually unbiased bases in dimension six: The four most distant bases, Phys. Rev. A 83, 062303 (2011).

[64] D. Goyeneche, Mutually unbiased triplets from non-affine families of complex Hadamard matrices in dimension 6, J. Phys. A 46, 105301 (2013).

[65] J. Batle, A. Farouk, M. Naseri, and M. Elhoseny, New approach to finding the maximum number of mutually unbiased bases in $\mathbb{C}^{6}$, Appl. Math. Inf. Sci. 10, 1 (2016).

[66] L. Chen and L. Yu, Mutually unbiased bases in dimension six containing a product-vector basis, Quantum Inform. Proc. 17, 198 (2018).
[67] M. Liang, M. Hu, Y. Sun, L. Chen, and X. Chen, Real entries of complex Hadamard matrices and mutually unbiased bases in dimension six, Linear Multilin. Algebra 69, 2908 (2021).

[68] D. McNulty and S. Weigert, All mutually unbiased product bases in dimension 6, J. Phys. A 45, 135307 (2012).

[69] D. McNulty and S. Weigert, On the impossibility to extend triples of mutually unbiased product bases in dimension six, Int. J. Quant. Inf. 10, 1250056 (2012).

[70] U. Haagerup, Orthogonal maximal abelian $*$-subalgebras of the $n \times n$ matrices and cyclic $n$-roots, Operator Algebras and Quantum Field Theory (Rome), Cambridge, MA: International Press, p. 296, (1996).

[71] W. Tadej and K. Życzkowski, A concise guide to complex Hadamard matrices, Open Syst. Inf. Dyn. 13, 133-177 (2006). and online version with W. Bruzda at http://chaos.if.uj.edu.pl/ ${ }^{\mathrm{karol} / \text { hadamard }}$

[72] T. Banica, Complex Hadamard matrices and applications, preprints hal-02317067 (2019) and ArXiv:1910.06911.

[73] F. Szöllösi, Complex Hadamard matrices of order 6: A four-parameter family, J. London Math. Soc. 85, 616 (2012).

[74] B. R. Karlsson, Three parameter complex Hadamard matrices of order 6, Lin. Alg. Appl. 434, 247 (2011).

[75] D. McNulty and S. Weigert, Isolated Hadamard matrices from mutually unbiased product bases, J. Math. Phys. 53, 122202 (2012).

[76] M. Saniga, M. Planat, and H. Rosu, MUBs and finite projective planes, J. Opt. B 6, L19 (2004).

[77] I. Bengtsson and A. Ericsson, MUBs and the complementarity polytope, Open Sys. Inf. Dyn. 12, 107 (2005).

[78] M. R. Kibler and M. Planat, A $S U(2)$ recipe for MUBs, Int. J. Mod. Phys. B 20, 1802 (2006).

[79] M. Planat, H. C. Rosu, and S. Perrine, A survey of finite algebraic geometrical structures underlying mutually unbiased quantum measurements, Found. Phys. 36, 1662 (2006).

[80] P. O. Boykin, M. Sitharam, P. H. Tiep, and P. Wocjan, MUBs and orthogonal decompositions of Lie algebras, Quant. Inform. Comput. 7, 371 (2007).

[81] T. Paterek, B. Dakić, and Č. Brukner, Mutually unbiased bases, orthogonal Latin squares, and hidden-variable models, Phys. Rev. A 79, 012109 (2009).

[82] T. Paterek, M. Pawłowski, M. Grassl, and C. Brukner, On the connection between mutually unbiased bases and orthogonal Latin squares, Phys. Scr. T140, 014031 (2010).

[83] M. N. Kolountzakis, M. Matolcsi, and M. Weiner, An application of positive definite functions to the problem of MUBs, Proc. Amer. Math. Soc. 146, 1143 (2018).

[84] G McConnell, H. Spencer, and A. Tahir, Evidence for and against Zauner's MUB conjecture in $\mathbb{C}^{6}$, preprint ArXiv:2103.08703.

[85] S. Weigert and M. Wilkinson, Mutually unbiased bases for continuous variables, Phys. Rev. A 78, 020303 (2008).

[86] D. S. Tasca, P. Sanchez, S. P. Walborn, and L. Rudnicki, Mutual Unbiasedness in Coarse-Grained Continuous Variables, Phys. Rev. Lett. 120, 040403 (2018).

[87] T. L. Silva, Ł. Rudnicki, D. S. Tasca, and S. P. Walborn, Periodic discretized continuous observables are neither continuous nor discrete, preprint ArXiv:2009.05062. 
[88] M. Appleby, I. Bengtsson, S. Flammia, and D. Goyeneche, Tight frames, Hadamard matrices and Zauner's conjecture, J. Phys. A 52, 295301 (2019).

[89] J. J. Sylvester, Thoughts on inverse orthogonal matrices, simultaneous signsuccessions, and tessellated pavements in two or more colours, with applications to Newton's rule, ornamental tile-work, and the theory of numbers, Phil. Mag. 34, 461 (1867).

[90] J. Hadamard, Résolution d'une question relative aux déterminants, Bull. Sci. Math. 17, 240 (1893).

[91] R. E. A. C. Paley, On orthogonal matrices, J. Math. Phys. 12, 311 (1933).

[92] H. Kharaghani and B. Tayfeh-Rezaie, A Hadamard matrix of order 428, J. Comb. Des. 13, 435 (2005).

[93] J. Rehacek, Z. Hradil, B. Stoklasa, M. Paúr, J. Grover, A. Krzic, and L. L. Sánchez-Soto, Multiparameter quantum metrology of incoherent point sources: Towards realistic superresolution, Phys. Rev. A 96, 062107 (2017).

[94] V. Ansari, B. Brecht, J. Gil-Lopez, J. M. Donohue, J. Rehacek, Z. Hradil, L. L. Sánchez-Soto, and C. Silberhorn, Achieving the Ultimate Quantum Timing Resolution, Phys. Rev. X Quantum 2, 010301 (2021).

[95] S. L. Braunstein and C. M. Caves, Statistical Distance and the Geometry of Quantum States, Phys. Rev. Lett. 72, 3439 (1994).

[96] V. Giovannetti, S. Lloyd, and L. Maccone, Quantum Metrology, Phys. Rev. Lett. 96, 010401 (2006).

[97] F. Albarelli, M. Barbieri, M. G. Genoni, and I. Gianani, A perspective on multiparameter quantum metrology: From theoretical tools to applications in quantum imaging, Phys. Lett. A 384, 126311 (2020).

[98] J. S. Sidhu and P. Kok, A geometric perspective on quantum parameter estimation, AVS Quantum Sci. 2, 014701 (2020).

[99] J. Liu, H. Yuan, X. Lu, and X. Wang, Quantum Fisher information matrix and multiparameter estimation, J. Phys. A 53, 023001 (2020).

[100] R. Demkowicz-Dobrzański, W. Górecki, and M. Guta, Multi-parameter estimation beyond quantum Fisher information, J. Phys. A 53, 363001 (2020).

[101] M. Szczykulska, T. Baumgratz, and A. Datta, Multiparameter quantum metrology, Adv. Phys. X 1, 621 (2016).

[102] Y. Yang, G. Chiribella, and M. Hayashi, Attaining the ultimate precision limit in quantum state estimation, Commun. Math. Phys. 368, 223 (2019).

[103] G. Toth and I. Apellaniz, Quantum metrology from a quantum information science perspective, J. Phys. A 47, 424006 (2014).

[104] J. Suzuki, Information geometrical characterization of quantum statistical models in quantum estimation theory, Entropy 27, 703 (2019).

[105] V. P. Belavkin, Generalized uncertainty relations and efficient measurements in quantum systems, Theoret. Math. Phys. 26, 213 (1976).

[106] S. Ragy, M. Jarzyna, and R. Demkowicz-Dobrzański, Compatibility in multiparameter quantum metrology, Phys. Rev. A 94, 052108 (2016).

[107] K. Matsumoto, A new approach to the Cramer-Rao-type bound of the pure-state model, J. Phys. A 35, 3111 (2002).
[108] J. Yang, S. Pang, Y. Zhou, and A. N. Jordan, Optimal measurements for quantum multiparameter estimation with general states, Phys. Rev. A 100, 032104 (2019).

[109] J. S. Sidhu, Y. Ouyang, E. T. Campbell, and P. Kok, Tight Bounds on the Simultaneous Estimation of Incompatible Parameters, Phys. Rev. X 11, 011028 (2021).

[110] M. Gessner, L. Pezze, and A. Smerzi, Sensitivity Bounds for Multiparameter Quantum Metrology, Phys. Rev. Lett. 121, 130503 (2018).

[111] L. Pezze, M. A. Ciampini, N. Spagnolo, P. C. Humphreys, A. Datta, I. A. Walmsley, M. Barbieri, F. Sciarrino, and A. Smerzi, Optimal Measurements for Simultaneous Quantum Estimation of Multiple Phases, Phys. Rev. Lett. 119, 130504 (2017).

[112] S. Razavian, M. G. A. Paris, and M. G. Genoni, On the quantumness of multiparameter estimation problems for qubit systems, Entropy 22, 1197 (2020).

[113] H. Kwon, K. C. Tan, T. Volkoff, and H. Jeong, Nonclassicality as a Quantifiable Resource for Quantum Metrology, Phys. Rev. Lett. 122, 040503 (2019).

[114] I. Kull, P. A. Guérin, and F. Verstraete, Uncertainty and trade-offs in quantum multiparameter estimation, J. Phys. A 53, 244001 (2020).

[115] J. Rubio and J. Dunningham, Quantum metrology in the presence of limited data, New J. Phys. 21, 043037 (2019).

[116] J. Rubio, P. Knott, and J. Dunningham, Non-asymptotic analysis of quantum metrology protocols beyond the Cramér-Rao bound, J. Phys. Commun. 2, 015027 (2018).

[117] X. Zhao, Y. Yang, and G. Chiribella, Quantum Metrology with Indefinite Causal Order, Phys. Rev. Lett. 124, 190503 (2020).

[118] A. Z. Goldberg, L. L. Sánchez-Soto, and H. Ferretti, Intrinsic Sensitivity Limits for Multiparameter Quantum Metrology, Phys. Rev. Lett. 127, 110501 (2021).

[119] F. Albarelli and R. Demkowicz-Dobrzanski, Probe incompatibility in multiparameter noisy quantum channel estimation, ArXiv:2104.11264.

[120] J. Friel, P. Palittapongarnpim, F. Albarelli, and A. Datta, Attainability of the Holevo-Cramér-Rao bound for twoqubit 3D magnetometry, ArXiv:2008.01502.

[121] F. Albarelli, J. F. Friel, and A. Datta, Evaluating the Holevo Cramér-Rao Bound for Multi-Parameter Quantum Metrology, Phys. Rev. Lett. 123, 200503 (2019).

[122] L. O. Conlon, J. Suzuki, P. Koy Lam, and S. M. Assad, Efficient computation of the Nagaoka-Hayashi bound for multiparameter estimation with separable measurements, npj Quantum Inf. 7, 110 (2021).

[123] R. F. Werner, Quantum states with Einstein-PodolskyRosen correlations admitting a hidden-variable model, Phys. Rev. A 40, 4277 (1989).

[124] R. Horodecki, P. Horodecki, M. Horodecki, and K. Horodecki, Quantum entanglement, Rev. Mod. Phys. 81, 865 (2009).

[125] The system is called $d \otimes d^{\prime}$ if and only if one associates with it the Hilbert space with a tensor-product structure, $\mathcal{H}_{d} \otimes \mathcal{H}_{d^{\prime}}$

[126] M. Horodecki, P. Horodecki, and R. Horodecki, Separability of mixed states: Necessary and sufficient conditions, Phys. Lett. A 223, 1 (1996). 
[127] Ł. Skowronek, There is no direct generalization of positive partial transpose criterion to the three-by-three case, J. Math. Phys. 57, 112201 (2016).

[128] H. Fawzi, The set of separable states has no finite semidefinite representation except in dimension $3 \times 2$, preprint ArXiv:1905.02575.

[129] P. Badziag, P. Horodecki, R. Horodecki, and R. Augusiak, Separability in terms of a single entanglement witness, Phys. Rev. A 88, 010301 (2013).

[130] P. Horodecki, Bound entanglement, in: D. Bruss, G. Leuchs (Editors) Quantum Information: From Foundations to Quantum Technology Applications, Wiley-VCH, (2019).

[131] W. Dür and H.-J. Briegel, Purification and distillation, in: D. Bruss, G. Leuchs (Editors) Quantum Information: From Foundations to Quantum Technology Applications, Wiley-VCH, (2019).

[132] C. H. Bennett, G. Brassard, S. Popescu, Sandu, B. Schumacher, J. A. Smolin, and W. K. Wootters, Purification of Noisy Entanglement and Faithful Teleportation via Noisy Channels, Phys. Rev. Lett. 76, 722 (1996).

[133] C. H. Bennett, D. P. DiVincenzo, J. A. Smolin, and W. K. Wootters, Mixed states entanglement and quantum error correction, Phys. Rev. A 54, 3824 (1996).

[134] D. Deutsch, A. Ekert, R. Jozsa, C. Macchiavello, S. Popescu, and A. Sanpera, Quantum Privacy Amplification and the Security of Quantum Cryptography Over Noisy Channels, Phys. Rev. Lett. 77, 2818 (1996).

[135] M. Horodecki, P. Horodecki, and R. Horodecki, Inseparable two Spin- $\frac{1}{2}$ Density Matrices can be Distilled to a Singlet Form, Phys. Rev. Lett. 78, 574 (1997).

[136] M. Horodecki and P. Horodecki, Reduction criterion of seperability and limits for a class of protocols of entanglement distillation, Phys. Rev. A 59, 4206 (1999).

[137] M. Horodecki, P. Horodecki, and R. Horodecki, MixedState Entanglement and Distillation: Is There a 'bound' Entanglement in Nature?, Phys. Rev. Lett. 80, 5239 (1998).

[138] P. Horodecki, Separability criterion and inseparable mixed states with positive partial transposition, Phys. Lett. A 232, 333 (1997).

[139] K. Życzkowski, Volume of the set of separable states II, Phys. Rev. A60, 3496 (1999).

[140] We now on purpose denote the Hilbert-space dimension by $d$, while in the former section we used $N$, in order to follow the notation common in the subfield.

[141] L. Clarisse, The distillability problem revisited, Quant. Inform. Comput. 6, 539 (2006).

[142] D. P. DiVincenzo, P. W. Shor, J. A. Smolin, B. M. Terhal, and A. V. Thapliyal, Evidence for bound entangled states with negative partial transpose, Phys. Rev. A 61, 062312 (2000).

[143] L. Pankowski, M. Piani, M. Horodecki, and P. Horodecki, A few steps more towards NPT bound entanglement, IEEE Trans. Inf. Theory 56, 4085 (2010).

[144] L. Clarisse, Entanglement Distillation. A Discourse on Bound Entanglement in Quantum Information Theory, (2006), ArXiv:quant-ph/0612072v1.
[145] W. Dür, J. I. Cirac, M. Lewenstein, and D. Bruss, Distillability and partial transposition in bipartite systems, Phys. Rev. A 61, 062313 (2000).

[146] T. Eggeling, K. G. H. Vollbrecht, R. F. Werner, and M. M. Wolf, Distillability via Protocols Respecting the Positivity of Partial Transpose, Phys. Rev. Lett. 87, 257902 (2001).

[147] G. Smith and J. Yard, Quantum communication with zerocapacity channels, Science 321, 1812 (2010).

[148] A. Müller-Hermes, D. Reeb, and M. M. Wolf, Positivity of linear maps under tensor powers, J. Math. Phys. 57, 015202 (2016).

[149] J. Watrous, Many Copies may be Required for Entanglement Distillation, Phys. Rev. Lett. 93, 010502 (2004).

[150] D. Doković, On two-distillable werner states, Entropy 18, 216 (2016).

[151] M. van der Eyden, T. Netzer, and G. De las Cuevas, Halos and undecidability of tensor stable positive maps, (2019), ArXiv:2110.02113.

[152] L. Qian, L. Chen, D. Chu, and Y. Shen, A matrix inequality related to the entanglement distillation problem, (2019), preprint ArXiv:1908.02428.

[153] R. A. Horn and C. R. Johnson, Topics on Matrix Analysis (Cambridge University Press, 1991).

[154] K. Horodecki, M. Horodecki, P. Horodecki, and J. Oppenheim, Secure key from Bound Entanglement, Phys. Rev. Lett. 94, 160502 (2005).

[155] M. Krenn, M. Erhard, and A. Zeilinger, Computerinspired quantum experiments, Nat. Rev. Phys. 2, 649 (2020).

[156] S. Bauml, M. Christandl, K. Horodecki, and A. Winter, Limitations on quantum key repeaters, Nat. Commun. 6, 6908 (2015).

[157] For a description of the rules for the open competition see the KCIK webpage [191], https://kcik.ug.edu.pl/post. php?id=1981.

[158] Posed in Ref. [192] and solved in Ref. [19].

[159] D. Goyeneche, Z. Raissi, S. Di Martino, and K. Życzkowski, Entanglement and quantum combinatorial designs, Phys. Rev. A 97, 062326 (2018).

[160] B. Musto and J. Vicary, Orthogonality for quantum latin isometry squares, EPTCS 287, 253 (2019).

[161] A. Rico, Absolutely maximally entangled states in small system sizes, Master Thesis, Innsbruck (2020).

[162] L. Euler, Recherches sur une nouvelle espece de quarres magiques, E530, (1782).

[163] Quantum version of bidding in bridge was analyzed in Ref. [193]. However, the cards used in this variant of the game were entirely classical, in contrast to the "quantum cards" required for the problem discussed here.

[164] J. Dénes and A. D. Keedwell, (eds.), Latin Squares: New Developments in the Theory and Applications, NorthHolland, Amsterdam, (1991).

[165] G. Tarry, Le Probléme des 36 officiers, Compte Rendu de 1'Association Française Pour l'Avancement des Sciences 2, 170 (1901).

[166] G. Perec, La Vie mode d'emploi, Hachette, 1978; English translation, Life: A User's Manual, Godline (1987).

[167] C. J. Colbourn and J. H. Dinitz, Mutually orthogonal Latin squares: A brief survey of constructions, J. Stat. Plan. Infer. 95, 9 (2001). 
[168] B. Musto and J. Vicary, Quantum Latin squares and unitary error bases, Quantum Inf. Comput. 16, 1318 (2016).

[169] N. Gisin and H. Bechmann-Pasquinucci, Bell inequality, Bell states and maximally entangled states for $n$ qubits, Phys. Lett. A 246, 1 (1998).

[170] A. Higuchi and A. Sudbery, How entangled can two couples get?, Phys. Lett. A 272, 213 (2000).

[171] V. M. Kendon, K. Nemoto, and W. J. Munro, Typical entanglement in multi-qubit systems, J. Mod. Opt. 49, 1709 (2002).

[172] A. J. Scott, Multipartite entanglement, quantum-errorcorrecting codes, and entangling power of quantum evolutions, Phys. Rev. A 69, 052330 (2004).

[173] I. D. K. Brown, S. Stepney, A. Sudbery, and S. L. Braunstein, Searching for highly entangled multi-qubit states, J. Phys. A 38, 1119 (2005).

[174] A. Borras, A. R. Plastino, J. Batle, C. Zander, M. Casas, and A. Plastino, Multi-qubit systems: Highly entangled states and entanglement distribution, J. Phys. A 40, 13407 (2007).

[175] P. Facchi, G. Florio, G. Parisi, and S. Pascazio, Maximally multipartite entangled states, Phys. Rev. A 77, 060304 R (2008).

[176] J. Martin, O. Giraud, P. Braun, D. Braun, and T. Bastin, Multiqubit symmetric states with high geometric entanglement, Phys. Rev. A 81, 062347 (2010).

[177] P. Facchi, G. Florio, U. Marzolino, G. Parisi, and S. Pascazio, Multipartite entanglement and frustration, NJP 12, 025015 (2010).

[178] L. Arnaud and N. Cerf, Exploring pure quantum states with maximally mixed reductions, Phys. Rev. A 87, 012319 (2013).

[179] W. Helwig, W. Cui, J. I. Latorre, A. Riera, and H. K. Lo, Absolute maximal entanglement and quantum secret sharing, Phys. Rev. A 86, 052335 (2012).

[180] F. Huber, O. Gühne, and J. Siewert, Absolutely Maximally Entangled States of Seven Qubits do not Exist, Phys. Rev. Lett. 118, 200502 (2017).
[181] F. Huber and N. Wyderka, Table of AME states at http://www.tp.nt.uni-siegen.de/+fhuber/ame.html.

[182] L. Clarisse, S. Ghosh, S. Severini, and A. Sudbery, Entangling power of permutations, Phys. Rev. A 72, 012314 (2005).

[183] D. Goyeneche, D. Alsina, J. I. Latorre, A. Riera, and K. Życzkowski, Absolutely maximally entangled states, combinatorial designs and multi-unitary matrices, Phys. Rev. A 92, 032316 (2015).

[184] F. Pastawski, B. Yoshida, D. Harlow, and J. Preskill, Holographic quantum error-correcting codes: Toy models for the bulk/boundary correspondence, J. High Energy Phys. 06, 149 (2015).

[185] F. Huber, C. Eltschka, J. Siewert, and O. Gühne, Bounds on absolutely maximally entangled states from shadow inequalities, and the quantum MacWilliams identity, J. Phys. A 51, 175301 (2018).

[186] M. Grassl, T. Beth, and M. Rötteler, On optimal quantum codes, Int. J. Quant. Inf. 2, 55 (2004).

[187] B. Musto, Constructing Mutually unbiased bases from quantum Latin squares, preprint ArXiv:1605.08919.

[188] P. Zanardi, Ch. Zalka, and L. Faoro, Entangling power of quantum evolutions, Phys. Rev. A 62, 030301 (2000).

[189] B. Jonnadula, P. Mandayam, K. Życzkowski, and A. Lakshminarayan, Entanglement measures of bipartite quantum gates and their thermalization under arbitrary interaction strength, Phys. Rev. Res. 2, 043126 (2020).

[190] X.-D. Yu, T. Simnacher, N Wyderka, H. C. Nguyen, and O. Gühne, Complete hierarchy for the quantum marginal problem, Nat. Commun. 12, 1012 (2021).

[191] Retrieved on 21 November (2021).

[192] List of Open Quantum Problems, Problem 35, IQOQI Vienna.

[193] S. Muhammad, A. Tavakoli, M. Kurant, M. Pawłowski, M. Żukowski, and M. Bourennane, Quantum Bidding in Bridge, Phys. Rev. X 4, 021047 (2014). 